ISSN: 1982-8373

\title{
PERFIL EPIDEMIOLÓGICO DAS MULHERES DO MUNICÍPIO DE VALENÇA COM LESÕES PRECURSORAS DO CÂNCER DE COLO DO ÚTERO
}

\author{
Epidemiological profile of women in the municipality of Valença with precursor \\ lesions of cervical cancer
}

\section{Camila Barbosa Ribeiro ${ }^{1}$ (1) Filomena Aste Silveira ${ }^{1}$ Juliana Monteiro Ramos Coelho ${ }^{1}$ (DJoão Alfredo Seixas ${ }^{1}$}

${ }^{1}$ Centro Universitário de Valença (UNIFAA) Valença $(R J)$

Autor correspondente:

João Alfredo Seixas

E-mail: jalseixas@gmail.com

\section{Como citar este artigo:}

RIBEIRO, C.B.; SILVEIRA, F. A.; COELHO, J.M.R., SEIXAS, J.A. Perfil epidemiológico das mulheres do Município de Valença com lesões precursoras do câncer de colo do útero. Revista Saber Digital, v. 14, n. 3, p. 30-42, 2021.

Data de Submissão: 29/10/21

Data de aprovação: $28 / 11 / 21$

Data de publicação: 21/12/21

\section{(ब) (1) $(9$}

Esta obra está licenciada com uma licença http://creativecommons.org/licenses/by-nc/4.0/

\section{RESUMO}

Objetivo: $O$ objetivo do presente estudo foi analisar o perfil epidemiológico de mulheres valencianas com lesões precursoras de câncer de colo do útero. Metodologia: Trata-se de um estudo descritivo de caráter quantitativo, realizado no período de junho de 2017 a agosto de 2018. Foram atendidas 6.430 mulheres na faixa etária estabelecida, com entrevistas analisando fatores de risco e as variáveis pré-definidas. Resultados: A faixa etária mais prevalente foi de 36 a 45 anos (36\%); Houve um predomínio da raça branca $(53,3 \%)$. Com relação a escolaridade, a maioria cursou entre 5 a 9 anos de estudo $(38,1 \%)$. A faixa de renda prevalece entre 1 a 2 salários mínimos (56\%). A maioria é casada $(47,3 \%)$ e com parceiro fixo $(76,2 \%)$. Grande parte possuem filhos $(82,7 \%)$, sendo de 2 a 3 a prole $(60,8 \%)$.A maioria das mulheres não usava método contraceptivo (53,3\%), sendo o anticoncepcional oral o mais usado (80\%). Quando perguntadas sobre IST, a maioria informou não ter sido infectadas $(78,7 \%)$. A maioria informou realizar o preventivo (96\%), com intervalo anual (48,6\%) e 77,3\% informou não ser tabagista. Conclusão: A análise do perfil epidemiológico e a investigação de outros fatores de risco tem contribuído muito no recrutamento da população de risco, permitindo a detecção precoce e avanços no tratamento da doença.

Palavras-chave: Saúde da Mulher, Câncer do colo do útero, Perfil epidemiológico.

\section{ABSTRACT}

Objective: The goal of this study wasto analyse the epidemiological profile of women from the city of Valença that present precursor injuries of cervical cancer. Methodology: It is a descriptive quantitative study, made from July 2017 until August 2018. It were seen 6430 women from a stablished age range and interviews to analyse risk factors and defined variables. Results: The age range with the bigest number of cases was from 36 to 45 years old $(36 \%)$. There was a predominance of white people $(53,3 \%)$. When it comes to education, most of them studied for 5 to 9 years $(38,1 \%)$. The income range that prevails is between 1 and 2 minimum wages (56\%). Most is married (46,3\%) and have a set partner (76,2\%). A big part has children $(82,7 \%)$, and most have between 2 and 3 children $(60,8 \%)$. Most women didn't used to use contraceptive methods $(53,3 \%)$, and the most used method was the oral contraceptive pill $(80 \%)$. When asked about STIs, most of them informed they wasn't infected $(78,7 \%)$. Most informed that make preventive exams $(96 \%)$, once a year $(48,6 \%)$ e $77,3 \%$ said they were not smokers. Conclusion: The analysis of the epidemiological profile and the investigation of other risk factors has been contributing a lot to the recruitment of the risk population, allowing and early diagnosis and advances in treatment.

Keywords: Women's Health,Uterine Cervical Neoplasms, Health Profile. 


\section{INTRODUÇÃO}

O câncer de colo do útero é o único tipo de câncer que possui uma vacina e um exame clínico que permite o seu diagnóstico precoce e o tratamento. Mesmo assim, ainda é um problema de saúde pública em vários países pelo mundo. No Brasil isso não é diferente, com maior incidência nas mulheres com vida sexual ativa, na faixa etária de 25 a 64 anos. Com aproximadamente 570 mil casos novos por ano no mundo, o câncer do colo do útero é o quarto tipo de câncer mais comum entre as mulheres. Ele é responsável por 311 mil óbitos por ano, sendo a quarta causa mais frequente de morte por câncer em mulheres (GÉRVAS; FERNANDEZ, 2016; WHO, 2020).

O câncer de colo do útero está associado à infecção persistente por subtipos oncogênicos do Papiloma Vírus Humano (HPV), especialmente o HPV16 e o HPV-18, responsáveis por cerca de $70 \%$ dos cânceres cervicais. Estimase que cerca de $80 \%$ das mulheres sexualmente ativas irão adquiri-lo ao longo de suas vidas. (BRASIL, 2017) A realização periódica do exame de colpocitologia oncótica continua sendo a estratégia mais adotada para o rastreamento do câncer do colo uterino, porém a detecção do HPV, vem se tornando parte das novas recomendações de triagem, resultando em grandes mudanças nas diretrizes (MITTELDOR, 2016).

Apesar das altas taxas de incidência e mortalidade, o câncer de colo uterino tem um tempo de evolução lento, o que permite ser facilmente diagnosticada e tratada. Os prestadores de cuidados devem desenvolver estratégias de prevenção, detecção precoce e intervenção para reduzir a crescente incidência de câncer de colo uterino. A triagem citológica a cada 3-5 anos reduz o câncer cervical cerca de 80\% (ELFGREN et al., 2017). O exame de câncer cervical envolve um processo complexo de citologia, papiloma vírus humano (HPV), colposcopia e uma infinidade de algoritmos para a identificação de pré-invasão, reduzindo marcadamente a morbidade e mortalidade por câncer cervical (LEES et al., 2016). 
O objetivo desse estudo é a identificação do perfil epidemiológico e a investigação de outros fatores de risco para o câncer de colo do útero, possibilitando o diagnóstico em uma fase inicial com porcentagens maiores de chances de cura.

\section{MATERIAL E MÉTODOS}

Este trabalho foi aprovado pelo comitê de ética e pesquisa com número CAAE: 74467317.0.0000.5246.

Foi realizado um estudo descritivo de abordagem quantitativa, onde se realizou entrevistas com as participantes, assim como uma avaliação física e clínica. O local de estudo foi o Ambulatório de Medicina Integrada (AMI) do Hospital Escola de Valença, localizado no município de Valença, no estado do Rio de Janeiro. O período de coleta de dados se deu de junho de 2017 a agosto de 2018. Participaram da pesquisa mulheres na faixa etária de 25 a 64 anos, que estiveram em consultas ginecológicas no referido ambulatório e apresentaram rastreio positivo para lesões precursoras do câncer de colo de útero. Durante a consulta de rotina a coleta de dados foi realizada através da apresentação de um questionário juntamente com o termo de consentimento a participar da pesquisa. As participantes responderam as perguntas referente a temática do estudo.

As perguntas do questionário compreendiam: faixa etária; raça; escolaridade; condição socioeconômica; situação conjugal; número de parceiros; paridade; se faz uso de contraceptivo; histórico de doença sexualmente transmissível; tabagismo; se realiza o preventivo, com que frequência e o local.

\section{RESULTADOS}

Foram atendidas no Ambulatório de Medicina Integrada (AMI) do Hospital Escola de Valença, 6.430 mulheres na faixa etária estabelecida de 25 a 64 anos no período referido do estudo, e destas, 150 apresentaram lesões precursoras 
de câncer de colo do útero, se tornando o objeto da pesquisa. A média de idade das participantes foi de 42 anos de idade e a faixa etária mais prevalente foi de mulheres de 36 a 45 anos (36\%), seguida de 26 e 35 anos $(28,7 \%)$ e apenas $2,7 \%$ das mulheres entrevistadas possuem mais de 65 anos.

Houve um predomínio de raça branca (53,3\%) seguido de pardas $(35,3 \%)$, pretas (10\%) e amarelas (1,3\%).

Em relação à escolaridade, a maioria das entrevistadas tinham de 5 a 9 anos de estudo $(38,1 \%)$, enquanto uma parcela pequena possui mais de 12 anos de estudo (17\%).

A faixa de renda prevaleceu entre 01 a 02 salários mínimos (56\%), o que demonstra um grande percentual de mulheres com renda baixa enquanto apenas um pequeno número de mulheres recebe mais de 05 salários mínimos $(0,7 \%)$.

A situação conjugal demonstrou que a maioria são casadas $(47,3 \%)$ e a minoria viúvas $(4,7 \%)$ e por isso, com relação ao número de parceiros, prevaleceu ser a maioria fixo $(76,2 \%)$, porém, um número expressivo de mulheres possui mais de 1 parceiro $(21,7 \%)$, um número alto comparando-se a quantidade de mulheres que não possuem parceiros.

A maioria das entrevistadas possuem filhos (82,7\%) e entre essas, a paridade prevaleceu na faixa entre 2 a 3 filhos (60,8\%). Mesmo com um número grande de mulheres que já possuem filhos, a maioria $(53,3 \%)$ não utilizava nenhum método para evitar a gravidez.

Dentre as que usavam (45,3\%), o método contraceptivo que prevaleceu foi o anticoncepcional oral (76,5\%).

Quando perguntadas sobre infecções sexualmente transmissíveis - IST, a maioria informou não terem sido infectadas $(78,7 \%)$, um número bem expressivo considerando o fato da maioria não utilizar nenhum método para evitar a gravidez $(53,3 \%)$ e um número alto de mulheres com mais de um parceiro $(21,7 \%)$. Dentre as que informaram já terem sido contaminadas, o HPV predominou com $60,7 \%$. 
Com relação ao preventivo, o resultado de mulheres que realização 0 exame foi de $96 \%$, com intervalo anual 48,6\% e com prevalência no AMI 45,1\%.

No que diz respeito ao tabagismo, a maioria informou não fazer uso $(77,3 \%)$ enquanto que ainda há uma parcela significativa que ainda faz uso de tabaco $(22,7 \%)$ mesmo sabendo dos malefícios.

A análise multivariada mostrou algumas variáveis independentes associadas importantes. Com relação aos anos de estudo, a maioria possuí de 05 a 09 anos apenas e dessas constatou-se associação negativa para realização de preventivo. Entretanto, esta associação foi estatisticamente significante apenas para o grupo de 05 a 09 anos, pois as mulheres que estavam na faixa de 10 a 12 anos de estudo, os resultados foram positivos com todas que realizaram o exame (Tabela 01$)$.

Tabela1 - Período de escolaridade das mulheres entrevistadas

\begin{tabular}{|c|c|c|c|c|}
\hline \multirow[t]{3}{*}{ Anos de estudo } & \multicolumn{4}{|c|}{ Realiza preventivo } \\
\hline & \multicolumn{2}{|c|}{ NÃO } & \multicolumn{2}{|l|}{ SIM } \\
\hline & $\mathrm{N}$ & $\%$ & $\mathrm{~N}$ & $\%$ \\
\hline Sem estudo & 1 & $20,0 \%$ & 2 & $1,4 \%$ \\
\hline De 1 a 4 & 1 & $20,0 \%$ & 22 & $15,8 \%$ \\
\hline De 5 a 9 & 3 & $60,0 \%$ & 52 & $37,4 \%$ \\
\hline De 10 a 12 & 0 & $0,0 \%$ & 38 & $27,3 \%$ \\
\hline Mais de 12 anos & 0 & $0,0 \%$ & 25 & $18,0 \%$ \\
\hline Total & 5 & $100,0 \%$ & 139 & $100,0 \%$ \\
\hline
\end{tabular}

O número de parceiros durante a vida permanece como importante causa para a aquisição de infecção sexualmente transmissível (IST). O que se observou é que mulheres com mais de um parceiro tem um risco relativo $>1$ $(R R=1,58)$ conferindo assim um fator de risco. Já possuir parceiro fixo se relacionou à proteção para a infecção com risco relativo $<1(R R=0,62)$. Porém, mais da metade das mulheres solteiras entrevistadas $(54,8 \%, n=17)$ e uma parcela das mulheres casadas $(19,4 \%, n=06)$, informaram ter mais de um parceiro, o que significa um número de mulheres muito grande e expostas ao risco de IST (Tabela 2). 
Perfil epidemiológico das mulheres do Município de Valença com lesões precursoras do câncer de colo do útero.

Ribeiro CB, Silveira FA, Coelho JMR, Seixas JÁ

Tabela 2 - relação de número de parceiros sexuais e incidência de ISTs

\begin{tabular}{lllll}
\hline Número de parceiros & \multicolumn{4}{l}{ Tem ou teve alguma IST } \\
\cline { 2 - 5 } & NÃO & SIM \\
\cline { 2 - 5 } & $\mathrm{N}$ & $\%$ & $\mathrm{~N}$ & $\%$ \\
\hline 1 parceiro fixo & 88 & $76,5 \%$ & 19 & $16,5 \%$ \\
\hline Mais de 1 parceiro & 21 & $18,3 \%$ & 8 & $7,0 \%$ \\
\hline Não tem parceiro & 6 & $5,2 \%$ & 1 & $0,9 \%$ \\
\hline Total & 115 & $100,0 \%$ & 28 & $24,3 \%$ \\
\hline
\end{tabular}

Não houve associação positiva para o tabagismo dentro da faixa etária prevalente com lesão precursora de câncer de colo uterino (36 a 45 anos). A maioria das mulheres entrevistadas $(77,3 \%, n=116)$ não fazem uso de tabaco. Dentre as tabagistas $(22,7 \%, n=36)$, a maioria se concentrou na faixa de 26 a 35 anos $(38,2 \%, n=13)$ (Tabela 3).

Tabela 3 - relação do tabagismo com a faixa etária

\begin{tabular}{|c|c|c|c|c|}
\hline \multirow[t]{3}{*}{ Faixa Etária } & \multicolumn{4}{|c|}{ Tabagista } \\
\hline & \multicolumn{2}{|l|}{ NÃO } & \multicolumn{2}{|c|}{ SIM } \\
\hline & $\mathrm{N}$ & $\%$ & $\mathrm{~N}$ & $\%$ \\
\hline De $18 a 25$ anos & 4 & $3,4 \%$ & 1 & $2,9 \%$ \\
\hline $\begin{array}{l}\text { De } 26 \text { anos a } 35 \\
\text { anos }\end{array}$ & 30 & $25,9 \%$ & 13 & $38,2 \%$ \\
\hline $\begin{array}{l}\text { De } 36 \text { anos a } 45 \\
\text { anos }\end{array}$ & 45 & $38,8 \%$ & 9 & $26,5 \%$ \\
\hline $\begin{array}{l}\text { De } 46 \text { anos a } 55 \\
\text { anos }\end{array}$ & 20 & $17,2 \%$ & 7 & $20,6 \%$ \\
\hline $\begin{array}{l}\text { De } 56 \text { anos a } 65 \\
\text { anos }\end{array}$ & 14 & $12,1 \%$ & 3 & $8,8 \%$ \\
\hline Acima de 65 anos & 3 & $2,6 \%$ & 1 & $2,9 \%$ \\
\hline Total & 116 & $100,0 \%$ & 34 & $100,0 \%$ \\
\hline
\end{tabular}




\section{DISCUSSÃO}

A identificação das lesões precursoras do câncer de colo de útero são de grande importância na prevenção secundária do câncer propriamente dito. Existem, no entanto, algumas variáveis que podem nos atentar para uma população de risco. Com isso, a amostra que compôs o presente trabalho foi dividia de acordo com idade, raça, anos de estudo, dentre outros. Através destas informações chegou-se aos resultados epidemiológicos expressos no quadro adiante (Quadro 1):

Quadro 1 - Relação das variáveis sociodemográficas encontradas no estudo

\begin{tabular}{|c|c|c|c|c|c|}
\hline $\begin{array}{l}\text { Variáveis } \\
\text { sociodemográficas }\end{array}$ & $\mathrm{n}$ & $\%$ & $\begin{array}{l}\text { Variáveis } \\
\text { sociodemográficas }\end{array}$ & $\mathrm{n}$ & $\%$ \\
\hline \multicolumn{3}{|l|}{ Idade } & \multicolumn{3}{|l|}{ Raça/Cor } \\
\hline De 26 anos a 35 anos & 43 & $28,7 \%$ & \multirow{2}{*}{ Branco } & \multirow{2}{*}{80} & \multirow{2}{*}{$53,3 \%$} \\
\hline De 36 anos a 45 anos & 54 & $36,0 \%$ & & & \\
\hline De 46 anos a 55 anos & 27 & $18,0 \%$ & Pardo & 53 & $35,3 \%$ \\
\hline De 56 anos a 65 anos & 17 & $11,3 \%$ & \multirow{3}{*}{ Amarelo } & 15 & $10 \%$ \\
\hline De 18 a 25 anos & 5 & $3,3 \%$ & & \multirow{2}{*}{2} & \multirow{2}{*}{$1,3 \%$} \\
\hline Acima de 65 anos & 4 & $2,7 \%$ & & & \\
\hline \multicolumn{3}{|l|}{ Renda Mensal } & \multicolumn{3}{|l|}{ Anos de estudo } \\
\hline 01 a 02 salários mínimos & 2 & $1,3 \%$ & $1 \mathrm{~A} 4$ & 3 & $2,0 \%$ \\
\hline 3 a 5 salários mínimos & 38 & $25,3 \%$ & $10 \mathrm{~A} 12$ & 23 & $15,6 \%$ \\
\hline $\begin{array}{l}\text { Mais de } 05 \text { salários } \\
\text { mínimos }\end{array}$ & 84 & $56,0 \%$ & 5 A 9 & 56 & $38,1 \%$ \\
\hline \multirow{3}{*}{$\begin{array}{l}\text { Menos que } 01 \text { salário } \\
\text { mínimo } \\
\text { Sem renda }\end{array}$} & \multirow[t]{2}{*}{24} & $16,0 \%$ & Mais de 12 anos & 38 & $25,9 \%$ \\
\hline & & $1,4 \%$ & \multirow{2}{*}{$\begin{array}{l}\text { Não informou } \\
\text { Nenhum }\end{array}$} & 25 & $17,0 \%$ \\
\hline & 02 & & & 5 & $3,4 \%$ \\
\hline \multicolumn{3}{|l|}{ Situação Conjugal } & \multicolumn{3}{|l|}{ Número de Parceiros } \\
\hline Casado & \multicolumn{2}{|c|}{$47,3 \%$} & 1 parceiro fixo & 7 & $4,9 \%$ \\
\hline Divorciado & 55 & $36,7 \%$ & Mais de 1 & 109 & $76,2 \%$ \\
\hline Solteiro & 17 & $11,3 \%$ & Não informou & 31 & $21,7 \%$ \\
\hline Viúvo & 7 & $4,7 \%$ & Não tem & 3 & $2,1 \%$ \\
\hline \multicolumn{3}{|l|}{ Possui Filhos } & \multicolumn{3}{|c|}{ Já teve/tem alguma IST } \\
\hline \multirow[t]{2}{*}{ Não } & 124 & $82,7 \%$ & Não & 118 & $78,7 \%$ \\
\hline & & & Sim & 28 & $18,7 \%$ \\
\hline Não informou & 23 & $15,3 \%$ & Não informou & 4 & $2,7 \%$ \\
\hline Sim & 3 & $2,0 \%$ & Se sim qual IST & & \\
\hline Quantos filhos & & & HPV & 17 & $60,70 \%$ \\
\hline & & & Verrugas & 5 & $17,90 \%$ \\
\hline
\end{tabular}


Ribeiro CB, Silveira FA, Coelho JMR, Seixas JÁ

\begin{tabular}{|c|c|c|c|c|c|}
\hline $\begin{array}{l}\text { Variáveis } \\
\text { sociodemográficas }\end{array}$ & $\mathrm{n}$ & $\%$ & $\begin{array}{l}\text { Variáveis } \\
\text { sociodemográficas }\end{array}$ & $\mathrm{n}$ & $\%$ \\
\hline Apenas 1 filho & 42 & $33,9 \%$ & Sífilis & 2 & $7,10 \%$ \\
\hline De 2 a 3 filhos & 63 & $50,8 \%$ & Bartholinite & 1 & $3,60 \%$ \\
\hline & & & Candidíase & 1 & $3,60 \%$ \\
\hline De 4 a 5 filhos & 12 & $9,7 \%$ & Gonorréia & 1 & $3,60 \%$ \\
\hline Mais de 5 filhos & 7 & $5,6 \%$ & VDRL & 1 & $3,60 \%$ \\
\hline \multicolumn{3}{|c|}{ Algum método para evitar gravidez } & \multicolumn{3}{|l|}{ Realiza preventivo } \\
\hline Não & 80 & $53,3 \%$ & Sim & 144 & $96,0 \%$ \\
\hline Sim & 68 & $45,3 \%$ & Não & 5 & $3,3 \%$ \\
\hline Não informou & 2 & $1,3 \%$ & Não informou & 1 & $0,7 \%$ \\
\hline \multicolumn{3}{|l|}{ Se sim qual } & \multicolumn{3}{|l|}{ Frequência do exame } \\
\hline Anticoncepcional & 52 & $76,5 \%$ & A cada 3 meses & 2 & $1,4 \%$ \\
\hline Preservativo & 12 & $17,6 \%$ & A cada 6 meses & 41 & $28,5 \%$ \\
\hline Outros & 3 & $4,4 \%$ & \multirow{2}{*}{ Uma vez ao ano } & \multirow[t]{2}{*}{70} & \multirow{2}{*}{$48,6 \%$} \\
\hline Não informou & 1 & $1,5 \%$ & & & \\
\hline \multicolumn{3}{|l|}{ Tabagismo } & A cada 2 anos & 13 & $9,0 \%$ \\
\hline Não & 116 & $77,3 \%$ & \multirow{2}{*}{$\begin{array}{l}\text { A cada } 3 \text { anos ou mais } \\
\text { Não informou }\end{array}$} & 9 & $6,3 \%$ \\
\hline Sim & 34 & $22,7 \%$ & & 9 & $6,3 \%$ \\
\hline
\end{tabular}

A partir da análise das variáveis, observou-se que a maior incidência de pacientes com lesões precursoras de neoplasia de colo de útero, ocorreu em mulheres brancas (53,3\%; $n=80)$, entre os 36 a 45 anos $(36 \% ; n=54)$, com baixo grau de escolaridade na faixa de 05 a 09 anos de estudo, ou seja, ensino fundamental completo ou inferior $(38,1 \% ; n=56)$, com renda mensal entre $01 \mathrm{e}$ 02 salários mínimos (56\%; $n=84)$ e com parceiro fixo $(76,2 \%, n=109)$.

Acredita-se que a maior incidência de câncer de colo uterino em mulheres de baixa escolaridade e de baixo nível socioeconômico deva-se, respectivamente, à falta de conhecimento, de acesso a informação e às dificuldades para chegarem aos serviços de saúde e aos programas de prevenção e tratamento eficazes (ROSA et. al., 2009).

Outro fator de risco importante que implica diretamente na maior exposição ao HPV que é a causa mais comum do câncer do colo uterino, é a quantidade de parceiros sexuais, independente de outros fatores de risco associados. A média de parceiros sexuais em mulheres portadores de neoplasia de câncer de colo uterino é igual a 4,2 (RAMA et. al., 2008). Do total analisado 
das pacientes $(n=150), 21,7 \%(n=31)$ possuíam mais de um parceiro, o que significa um número muito alto principalmente pelo fato de que mais da metade $(53,3 \%, n=80)$ das mulheres não usam métodos contraceptivos e dentre as que tiveram IST(18,7\%, n=28), o HPV foi o destaque com $60,7 \%$ dos casos. Um parceiro fixo apenas relacionou-se à proteção para a infecção, comparado às mulheres que não referiram esta condição, conforme observado em estudos anteriores (HERRERO, et al., 2005).

Estudos realizados na América Latina detectaram associação entre 0 risco de câncer cervical e os seguintes hábitos sexuais dos parceiros sexuais masculinos: contato sexual com número elevado de parceiras, relações sexuais extraconjugais e relações sexuais com profissionais do sexo (ELUF-NETO; NASCIMENTO, 2001).

Quando a incidência de lesões precursoras do câncer do colo uterino é avaliada por faixa etária, as mulheres entre 26 e 35 anos apresentaram a segunda maior incidência $(28,7 \%, n=43)$, coincidindo com a observação de outros autores (RAMA et al., 2006).

Esse dado corrobora o que se conhece sobre a história natural da doença, mostrando maior infecção pelo HPV após o início da atividade sexual. Entretanto, para mulheres com 30 anos ou mais, que foi a prevalência desse estudo (36\%, $\mathrm{n}=54$ ), a positividade para lesões precursoras pode indicar persistência da infecção por HPV, o que demandaria seguimento mais cuidadoso e constante (RAMA et al., 2008).

Com relação ao exame de preventivo, a maioria das pacientes (96\%, $\mathrm{n}=144$ ) informou realizar a coleta anual de acordo com as diretrizes de rastreamento do Ministério da Saúde (MS). Porém apesar de todos os programas de prevenção oferecidos pelo governo, ainda persiste um grande contingente de mulheres que não têm acesso ou desconhecem a necessidade de realizar os exames preventivos periódicos (LIMA et al., 2011).

No que se refere a variável raça/cor, foi observada associação estatisticamente significativa entre a coloração da pele e as anormalidades do exame citológico, sendo a cor branca a que apresentou maior frequência dessas 
alterações na amostra estudada $(53,3 \%, \mathrm{n}=80)$, achado controverso ao observado no rastreamento feito em outro estudo (RAMA et al., 2008) que identificou uma prevalência maior em mulheres negras. Isso justifica-se pelo fato de que o n desse grupo (cor de pele negra) foi o segundo menor obtido para a variável cor $(10 \%, n=15)$, e que podemos estar diante de uma baixa demanda de mulheres negras nesse tipo de serviço. Uma possível explicação seria a maior proporção de não realização do exame citopatológico por negras, evidenciado em um estudo desenvolvido em Pelotas-RS (FERREIRA et al., 2011).

Vários estudos apontam a realização do exame preventivo como de grande importância ao diagnóstico das lesões e como forma de controle do câncer de colo uterino (FERREIRA et al., 2011; BEKKERS et al., 2004; LEDWABA et al., 2004). Nossa amostra não evidenciou dados significativos referentes às lesões precursoras de colo uterino quando a variável em questão foi arealização ou não do preventivo e a periodicidade (3 meses, 06 meses, uma vez ao ano, a cada 02 anos e a cada 03 anos ou mais). Quase todas as mulheres entrevistadas relataram realizar o exame de acordo com o rastreio ( $96 \%, \mathrm{n}=144)$. Pensamos que esse resultado exija uma análise criteriosa, levando-se em consideração que há uma lacuna grande de mulheres que não realizam o exame e se trata de um público ainda desconhecido e que muitas dessas mulheres foram encaminhadas para o Serviço de Ginecologia do AMI devido a uma alteração detectada no último exame citológico realizado nas Estratégias de Saúde da Família ou Casa de Saúde da Mulher. Logo, a resposta a tal questionamento não retrataria a situação anterior ao achado alterado.

A prevalência de doenças sexualmente transmissíveis foi significativa em nosso estudo $(18,7 \%, n=28)$, porém não houve associação estatística significante entre história de IST e presença de lesões precursoras de câncer de colo uterino, visto que a maioria $(78,7 \%, \mathrm{n}=118)$ que apresentaram lesão, nunca tiveram IST. Entretanto, Ferreira (2011) e Rama et al. (2008) relataram que mulheres com história de doença sexualmente transmissível apresentaram chance maior de tais alterações em relação a aquelas que negaram esta condição. A presença do HPV dentre as que relataram IST foi o destaque 
(60,7\%, n=17). Pensamos que a ausência de história de DST em nossa amostra,baseado apenas no relato da entrevistada,possa não refletir uma situação real, seja por desconhecimento ou pudor da paciente ao responder ou mesmo uma falha do entrevistador.

A associação entre uso de métodos contraceptivos e maior propensão alesões precursoras de câncer de colo uterino, apresentou significância estatística em nosso estudo, contrariando outros estudos (FERREIRA et al., 2011; RAMA et al., 2008). Nossa pesquisa evidenciou que pode haver uma relação de alterações citológicas no exame com o não uso de métodos contraceptivos uma vez que mais da metade $(53,3 \%, n=80)$ das mulheres que apresentaram lesões, não usavam métodos contraceptivos e as que usavam $(45,3, n=68)$, usavam em sua maioria apenas anticoncepcional oral (ACO) (76,5\%, $n=52)$, o que previne apenas a gravidez e não protege das ISTs, principalmente o HPV que foi o mais relatado.

Em relação à variável paridade, o estudo não evidenciou relação entre lesões precursoras de câncer de colo uterino e número de gestações. Essa associação é ainda divergente na literatura científica, havendo estudos que relatam uma associação diretamente proporcional entre neoplasia de cérvice e número de gestações, outros que evidenciam maior associação entre lesão de colo uterino e mulheres que nunca gestaram e alguns que não encontraram nenhuma associação (FERREIRA et al., 2011).

\section{CONCLUSÃO}

Este estudo apresentou uma descrição sobre algumas variáveis epidemiológicas de mulheres com lesões precursoras do câncer do colo uterino na tentativa de despertar o interesse e a atenção dos cuidadores dessa população quanto às formas de prevenção dessa patologia.

Houve diminuição de algumas variáveis epidemiológicas, o que pode ser reflexo dos investimentos nas ações de prevenção e controle de fatores de risco do câncer do colo uterino principalmente na fase sexual ativa a qual evidenciou maior prevalência na pesquisa 


\section{REFERÊNCIAS}

BEKKERS, RLM; MASSUGER, L.F.A.G.; BULTEN, J.; MELCHERS, W.J.G. Epidemiological and clinical aspects of human papillomavirus detection in the prevention of cervical cancer. Reviews in Medical Virology, v.14, no. 2, p. 95105, 2004.

BRASIL, Ministério da Saúde. Instituto Nacional de Câncer José Alencar. Controle do câncer do colo do útero: fatores de risco, Brasília: Ministério da Saúde, 2017. Disponível em: https://www.inca.gov.br/controle-do-cancer-docolo-do-utero/fatores-de-risco. Acesso em: 14 jun. 2021.

ELFGREN, K.; ELFSTRO, M.K.M.; NAUCLER, P., ARNHEIM-DAHLSTRÖM, L.; DILLNER, J. Management of women with human papilloma virus persistence: long-term follow-up of a randomized clinical trial. Am J ObstetGynecol, v. 216, n. 264, p. 1-7, 2017.

ELUF-NETO, J.; NASCIMENTO C.M. Cervical cancer in LatinAmerica. Semin Oncol, v. 28, n. 2, p. 188-197, 2001.

FERREIRA, A.S.S., et al. Aspectos clínico-epidemiológicos das pacientes portadoras de alterações colpocitologicas atendidas no Hospital Universitário da UFJF. HU Revista, v. 37, n. 4, p. 421-429, 2011.

GERVÁS, J.; FERNANDEZ, M.P. São e salvo: e livre de intervenções médicas desnecessárias. Porto Alegre: Artmed, 2016.

HERRERO R., et al. Epidemiologic profile of type-specifi c human papillomavirus infection and cervical neoplasia in Guanacaste, Costa Rica. J Infect Dis, v. 191, n. 11, p. 1796-1807, 2005.

LEDWABA, T; DLAMINI, Z; NAICKER, S; BHOOLA, K. Molecular genetics of human cervical cancer: role of papillomavirus and the apoptotic cascade. Biological Chemistry, Johannesburg, v. 385, no. 8, p. 671-682, Aug. 2004

LEES, B.F.; ERICKSON, B.; HUH, W.K. Cervical câncer screening evidence behind the guidelines. American Journal of Obstetrics Gynecology, v. 212., n. 4, p. 438-446, abr. 2016.

LIMA, MEA; OLIVEIRA, ASM; CABRAL, CN; COSTA, JS; MAYARA, MMH; BARBOSA, GAAL. Perfil epidemiológico das pacientes com câncer de colo 
uterino atendidas no serviço de cancerologia da fundação assistencial da Paraíba em Campina Grande. Revista saúde \& ciência, v. 2, n.1, p. 89-93 2011.

MITTELDORF, C.A.T.S. Cervical câncer screening: from Papsmearto future strategies. J Bras Patol Med Lab., v. 52, n. 4, p. 238-245, 2016.

RAMA, C.H., et al. Prevalência do HPV em mulheres rastreadas para o câncer cervical. Rev Saúde Pública, v. 42, n. 1, p. 123-130, 2008.

RAMA, C.H., et. al. Detecção sorológica de anti HPV 16 e 18 e sua associação com achados do Papanicolau em adolescentes e mulheres jovens. Rev Assoc Med Bras, v. 52, n. 1, p. 43-47, 2006.

ROSA, M.I., et al. Papilomavírus humano e neoplasia cervical. Cad. Saúde Pública, v, 25, n.5, p.953-964, 2009.

WORLD HEALTH ORGANIZATION. Comprehensive cervical cancer prevention and control: a healthier future for girls and woman. Disponível em: https://apps.who.int/iris/bitstream/handle/10665/78128/9789241505147 eng.pdf ;isessionid=CC278A385C4C002D94AECB3950F3C82E? sequence $=3$. Acesso em: 10/02/2020. 